\title{
Modelling and Detection of Turn-to-Turn Faults in Transformer Windings Using Finite Element Analysis and Instantaneous Exciting Currents Space Phasor Approach
}

\author{
Malihe Abi, Mohammad Mirzaie* \\ Faculty of Electrical and Computer Engineering, Babol Noshirvani University of Technology, Babol, Iran
}

\begin{abstract}
In this paper, a novel method based on online monitoring of Instantaneous Exciting Current Space Phasor is presented in order to detect inter-turn faults on the transformer windings. This approach detects winding faults by comparison between presentation of the Instantaneous Exciting Current Space Phasor under healthy and faulty condition. In this work, the angular speed of Instantaneous Exciting Current Space Phasor has been introduced as one of the fault detection tools that has good sensitivity for detection of minor inter-turn faults. Firstly, a typical transformer is simulated based on Finite Element Analysis (FEA) to investigate the transformer behavior under different conditions. Then, the accuracy and performance of proposed diagnosis technique are studied by applying it to the simulated transformer.
\end{abstract}

Index Terms: Angular speed, Finite Element Analysis, Instantaneous Exciting Current Space Phasor approach, Transformer, Turn-to-turn fault.

(C) 2014 Published by MECS Publisher. Selection and/or peer review under responsibility of the Research Association of Modern Education and Computer Science.

\section{Introduction}

Transformers are one of the most important and expensive devices in electrical systems that are critical links between the generation stations and consumers. Variety of unusual conditions and faults can be affected the transformers. Unplanned repairs such as fix or replacement of the faulty transformer are very costly and time consuming. One of the most sensitive parts of the transformer is the insulation system that can be exposed by electrical, mechanical and thermal stresses and moisture. Degradation of insulation system causes a breakdown in the insulation and leads to development the inter-turn faults. Internal turn-to-turn faults are the most difficult types of faults to detect within the transformers. If turn-to-turn fault has not been rapidly

* Corresponding author. Tel.: 01113210977; fax: 01113239214

E-mail address: sneha2bandekar@gmail.com 
detected, it can develop into more critical and costly to repair faults such as phase-to-phase or phase-to-ground faults. Therefore, quick detection of turn-to-turn faults is essential in order to protect the entire of electrical system and reduce the damage and repair cost. In this way, development of online techniques for condition monitoring and in order to diagnose of inter-turn faults is very important for improvement of the system reliability.

The advanced methods such as Wavelet Transforms [1, 2], S Transform [3, 4] and Hilbert Transform have been already applied for turn-to-turn fault detection in the transformer windings. Although these approaches detect the minute faults, but they are based on the signal processing and have complicated computation. Hence, these methods require large number of processors and instruments and so implementation of them is difficult [5].

In recent years, new techniques based on the Park Vector [6 - 11], Clarke Transform [12,13] and Space Phasor have been attended because of their simplicity and sensitivity in winding faults detection. In [6, 7] the inter-turn faults have been diagnosed by using supply current Park's Vector pattern. The fault diagnosis is based on recognizing the appearance of an elliptic pattern, related to the transformer supply current park's Vector representation. The ellipticity of pattern enlarges with the expansion of fault and whose major axis orientation is associated to the faulty phase. However, this approach is affected by the transformer load condition and it is difficult to distinguish between unbalanced load and winding fault cases. To overcome this problem, a method was proposed which is based on the analysis of the on-load exciting current Park's Vector pattern, and hence is not affected by the load condition of transformer [8 - 10]. Additionally, this technique improves the fault diagnosis sensitivity, as compared to the previous diagnosis approach. In [11] the extended Park's Vector approach has been applied to the differential currents and the severity factor is used to identify inter-turn faults and also discriminate between fault cases and inrush current condition.

In [12] the three phase currents of the transformer are converted to the modal current components using Clarke's transform and the transient modal currents from the both sides of the transformer are applied to the Fault Discrimination equation to distinguish between the internal faults and external faults from the polarity of its output.

Up to now, application of Space Phasor approach has been investigated for fault diagnosis in electrical machines by applying it to the terminal currents. In [14] the stator winding faults in a typical induction motor have been detected by monitoring of Instantaneous Stator Currents Space Phasor. However, the terminal currents are not affected by low level internal faults and consequently, this method is not sensitive sufficiently. It seems that applying the Space Phasor method to the exciting currents of the transformer will create a useful fault diagnosis approach that will very sensitive and independent of load condition and also it is stable in the external fault cases. Accordingly, this paper suggests the Instantaneous Exciting Current Space Phasor technique to identify turn-to-turn faults in the transformer windings. The characteristics of the Instantaneous Exciting Current Space Phasor (such as modulus, angle and angular speed) make change in a faulty transformer as compared to its healthy condition and hence, we propose to use these characteristics as fault detection tools. Firstly, a three phase transformer under normal operation and also during inter-turn fault condition has been simulated based on Finite Element Analysis in Maxwell 14.0 software. Then, the IECSP approach has been implemented in Matlab software and its validation has been examined by applying it to the Maxwell outputs. Finally, the fault detection tools of the proposed technique have been presented and related indexes have been compared together for assessment to the optimum indicator.

\section{Finite Element Model of the Transformer}

In order to simulate a turn-to-turn fault on the transformer winding, the defective transformer is modeled as shown in Fig 1. As seen from the figure, when a turn-to-turn short-circuit occurs on the transformer, the affected winding is divided in two subwindings " $a$ " and " $\mathrm{b}$ ", that are associated to the healthy and faulty part, respectively [10]. 

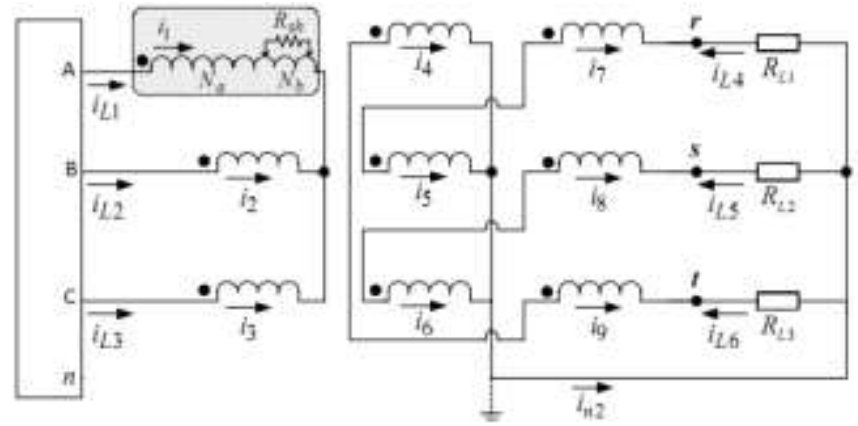

Fig. 1. Equivalent circuit for turn-to-turn fault in transformer winding

In this figure, the fault has been illustrated on the primary winding of phase A by connecting the fault resistance $\left(R_{s h}\right)$ across the shorted turns. The severity of fault depends on the number of shorted turns as well as the circulating current flowing of them that is limited by the fault resistance.

To investigate the transformer behavior in different situations, a two winding three phase transformer has been simulated in Maxwell 14.0 software which is based on Finite Element Analysis. The Finite Element Analysis is a quick and efficient way in simulation of modern engineering systems that solves a problem by separating the problem field into several elements and then implementing physical rules to each minor element.

Fig 2 shows the Finite Element Model of aforesaid transformer in geometric domain of Maxwell software.

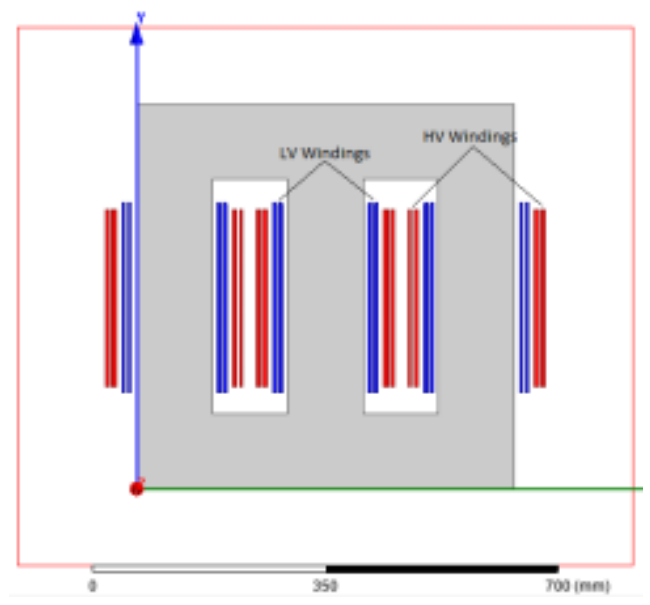

Fig. 2. FEM of transformer in Maxwell environment

Under healthy condition, each one of the transformer coils has been drawn as two whole regions and the nominal ampere-turn has been assigned to each region. For faulty transformer, the defective part of winding has been modeled as separated geometric region and desired value of ampere-turn has been allotted to it. Then, the Dirichlet boundary condition has been applied to the boundary surrounding the transformer. In order to modeling the nonlinear nature of transformer core, the magnetization curve of core material has been assigned to it. Afterwards, the circuit domain related to geometric domain has been designed in Ansoft Maxwell Circuit 
Editor environment. In the circuit domain, each separated region of coils in geometric domain has been modeled by its equivalent inductance calculated by software and the resistance corresponding to it.

In order to simulate the inter-turn fault, the geometric domain should be modified as well as circuit domain. In the equivalent circuit a time-controlled switch has been used in series with the fault resistance to create the winding fault in desired moment.

Finally, the simulated transformer has been analyzed by selecting the Transient mode for solution type and $100 \mu \mathrm{s}$ for time step (equal to $10 \mathrm{KHz}$ sampling frequency). The rated values of simulated transformer have been presented in Table 1.

Table 1. The rated values of the transformer

\begin{tabular}{ll}
\hline Transformer type & 3-Phase, 2-Winding \\
\hline Rated voltage ratio & 20/0.4 KV \\
\hline Rated apparent power & $100 \mathrm{KVA}$ \\
\hline Rated frequency & $50 \mathrm{HZ}$ \\
\hline Connection style & Yzn5 \\
\hline Turn ratio & $2944 / 68$ \\
\hline Core steel type & M5 \\
\hline
\end{tabular}

\section{Description of Transformer Behavior Under Faulty Condition}

The waveforms of the terminal currents as well as circulating current in the shorted turns that have achieved by FEA simulation are shown in Fig 3 and Fig 4. The transformer has been supplied from the HV side by the rated nameplate voltage and the rated resistive load has been connected to the LV side. A turn-to-turn fault involving $3 \%$ of the turns has been located on the primary and secondary windings of the simulated transformer, respectively. Also, the fault impedance value has been adjusted near to zero to create a metal-to-metal contact.

According to the Faraday's law, the input flux into a turn depends on the electro motive force (EMF) on its terminals for certain frequency and hence, the fundamental magneto motive force (MMF) of the transformer winding is fixed by the fixed EMF of voltage supply. As a result, when an inter-turn fault happens on the primary or the secondary winding, a great circulating current flows in the shorted turns to generate an opposite MMF to the fundamental MMF of winding and change the input flux into the shorted turns proportional to the decreased voltage across them. So, for the faults containing a minor fraction of the coil, the circulating current will be larger to be able to oppose the fundamental MMF of the coil as a result of high ratio of transformation between the whole winding and affected turns [15]. 


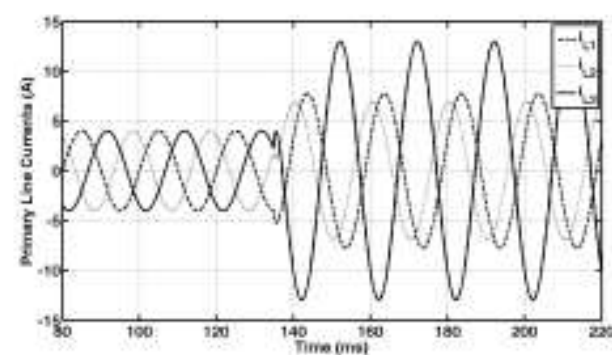

(a)

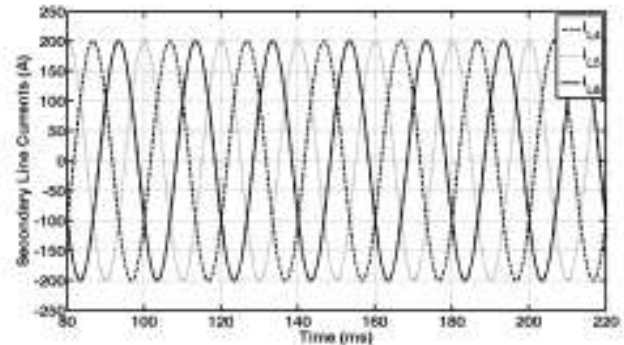

(b)

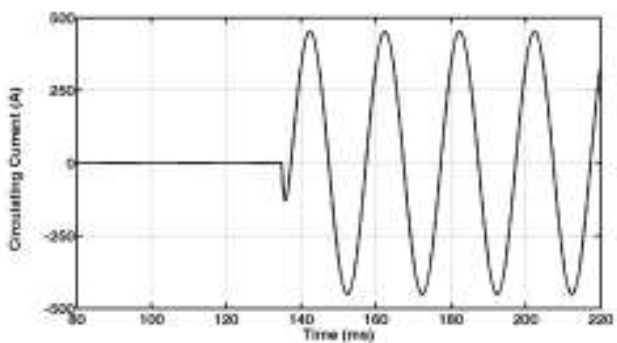

(c)

Fig. 3. Terminal currents and circulating current waveforms for a turn-to-turn fault involving $3 \%$ of winding on phase $\mathrm{C}$ of primary side

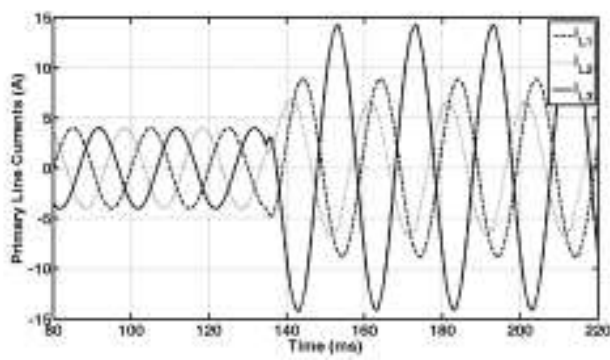

(a)

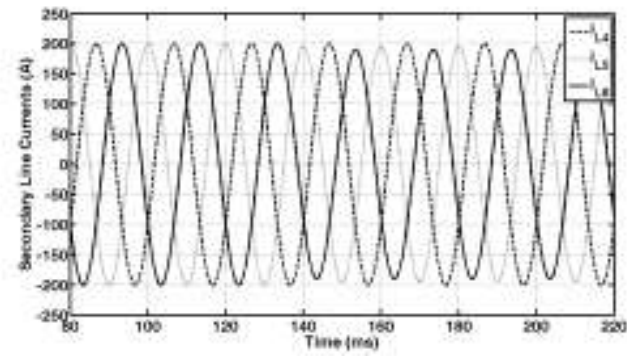

(b)

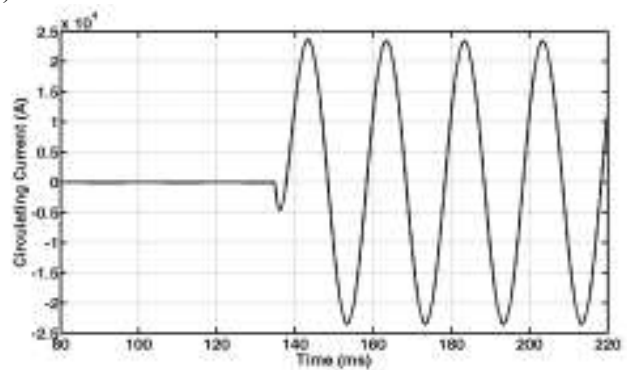

(c)

Fig. 4. Terminal currents and circulating current waveforms for a turn-to-turn fault involving $3 \%$ of winding on phase $\mathrm{C}$ of secondary side

When a turn-to-turn fault is located on the each one of transformer windings, since the fundamental MMF in the affected turns has been reduced, in order to preserve the fundamental MMF of whole winding, a higher 
current flows in the primary winding as shown in Fig 3 and Fig 4. It should be noted that the fault occurrence on the primary winding has a cumulative effect in decrement of the fundamental MMF and consequently, increment of the primary current to compensate it. Because there are lower turns in the primary side to generate the same MMF that demanded to preserve the fundamental flux of the transformer in this case. Furthermore, it can be mentioned that the induced voltages in the secondary side and so the output currents do not change because of the same fundamental MMF before and after the fault occurrence in the primary winding. While in the case of inter-turn short-circuit on the secondary side it is observed that the secondary terminal values is reduced as a result of the lessened effective number of the turns on the secondary winding [15].

\section{Concept of Instantaneous Current Space Phasor}

Two dimensional presentation of three phase variables as Instantaneous Current Space Phasor (ICSP) can be used to examine their balancing situation. The transformer ICSP as a function of instantaneous line currents for a system with positive sequence is defined by:

$$
\bar{i}=i_{L 1}+a i_{L 2}+a^{2} i_{L 3} ; a=e^{j \frac{2 \pi}{3}}
$$

Although (1) is similar to the expression related to the positive sequence component in the Fortesque Transform, but in order to calculate the ICSP the instantaneous values containing harmonic components is inserted in (1) instead of phasor values related to a given frequency.

We can write $\overline{\boldsymbol{i}}$ in Cartesian coordinates as below:

$$
\bar{i}=i_{\alpha}+j i_{\beta}
$$

Also, the ICSP is shown in polar coordinates by the following phrase:

$$
\bar{i}=m e^{j \theta}
$$

In the above phrase $m$ and $\theta$ are modulus and angle of the ICSP, respectively:

$$
\begin{aligned}
& m=\sqrt{i_{\alpha}{ }^{2}+i_{\beta}{ }^{2}} \\
& \theta=\arccos \left(i_{\alpha} / m\right)
\end{aligned}
$$

The angular speed of the ICSP can be defined as:

$$
\omega=d \theta / d t
$$

The ICSP locus is obtained by drawing $i_{\beta}$ in terms of $i_{\alpha}$. In normal situation, the terminal currents are balanced and the ICSP locus is a circle in the center of the coordinates plate. When an inter-turn fault is occurred on the each one of the transformer windings, the primary currents will be unbalanced and hence, the ICSP locus will be converted to an elliptic that its major axis orientation indicates the faulty phase and its ellipticity increases by the fault expansion. But, the terminal currents of the transformer are also unbalanced in 
presence of the unbalanced loads and external fault cases. So, the ICSP of the terminal currents is not suitable way for the fault diagnosis.

To conquer the aforesaid difficulty, a modified detection method according to the analysis of the Instantaneous Exciting Current Space Phasor (IECSP) locus is suggested. The three phase exciting currents of the transformer are balanced in the sound situation and also they are independent from condition in out of the transformer such as load condition and external faults. So, the proposed technique will be robust in varies load condition and external fault cases. The occurrence of winding faults leads to create an unbalanced system of the exciting currents that can be used as one of the diagnostic tools. Additionally, the exciting currents are more sensitive to the fault expansion and hence, this technique enhances the fault diagnosis sensitivity as compared to the former diagnosis approach. The IECSP technique will be employed for fault detection in the further section.

\section{Detection of Winding Faults Using Instantaneous Exciting Current Space Phasor Approach}

The transformer IECSP is achieved by the following expression:

$$
\bar{i}=i_{\text {exc } 1}+e^{j \frac{2 \pi}{3}} \cdot i_{\text {exc } 2}+e^{j \frac{4 \pi}{3}} \cdot i_{\text {exc } 3}
$$

In the above equation, $i_{\text {exc } 1}, i_{\text {exc } 2}$ and $i_{\text {exc } 3}$ are the exciting currents of phase $\mathrm{A}, \mathrm{B}$ and $\mathrm{C}$, respectively. The exciting currents equations of the transformer can be obtained based on the ampere-turns balance principle and they will different in various connections of the transformer. The exciting currents equations for the Yzn5 connection (notation as per Fig 1) will be:

$$
\left[\begin{array}{l}
i_{\text {exc } 1} \\
i_{\text {exc } 2} \\
i_{\text {exc } 3}
\end{array}\right]=\left[\begin{array}{l}
i_{L 1} \\
i_{L 2} \\
i_{L 3}
\end{array}\right]+\left(\frac{N_{2} / 2}{N_{1}}\right) \cdot\left[\begin{array}{rrr}
-1 & 0 & 1 \\
1 & -1 & 0 \\
0 & 1 & -1
\end{array}\right]\left[\begin{array}{l}
i_{L 4} \\
i_{L 5} \\
i_{L 6}
\end{array}\right]
$$

\subsection{Study of fault detection tools}

The following items can be considered as the fault detection tools by comparing them in before and after the fault occurrence:

- The exciting current of the faulty phase,

- The IECSP locus,

- The IECSP modulus,

- The IECSP angular speed.

In this section, the above-mentioned fault detection tools are studied and discussed, comprehensively. In the entire of fault cases presented in this section, the fault resistance has been adjusted so that limit the current in the defective part of the winding to 1.25 of rated current in the secondary side.

Under normal condition, the exciting currents are slightly unbalanced and non-sinusoidal due to the asymmetry and nonlinearity of the transformer core. Consequently, the IECSP locus will be an asymmetric hexagon in healthy transformer. The occurrence of the inter-turn short-circuits on each side of the transformer causes an increment in amplitude of the exciting current corresponding to the affected phase and leads to a deformed IECSP locus.

Fig 5 shows the three phase exciting currents and the IECSP modulus corresponding to the healthy condition as well as faulty situation with various fault severities in phase $\mathrm{C}$ of the secondary side. It is observed that the 
exciting current amplitude of the faulty phase is increased more than other phases with the fault extension and also the extremum values of IECSP modulus change obviously.

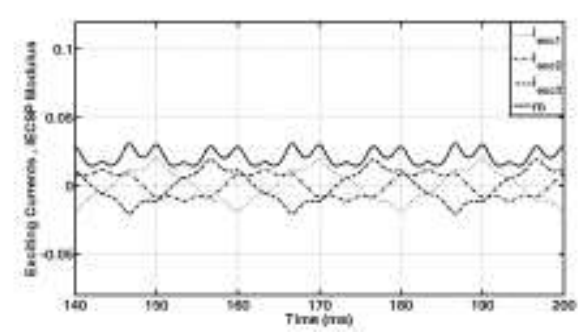

(a)

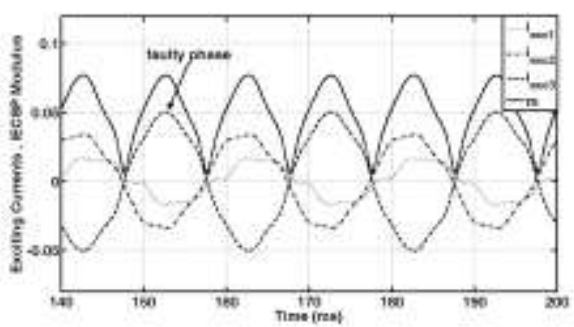

(c)

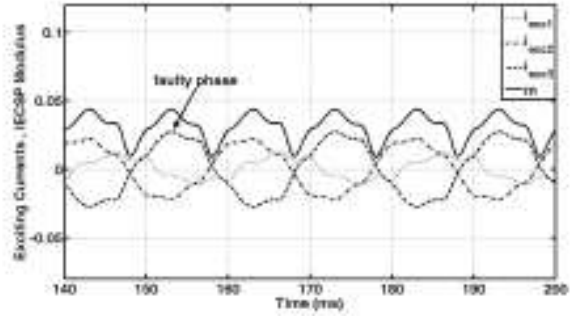

(b)

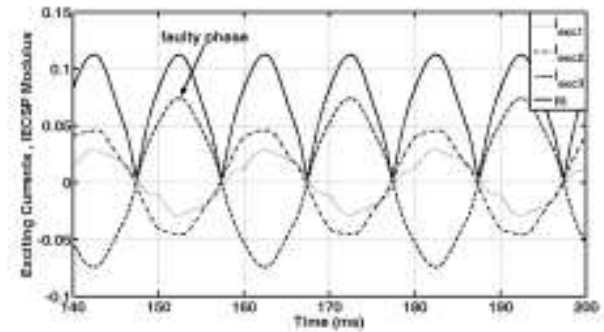

(d)

Fig. 5. Exciting currents and IECSP modulus waveforms for: (a) healthy condition; and for a turn-to-turn fault involving: (b) $3 \%$ (2 turns); (c) $6 \%$ (4 turns); (d) $9 \%$ (6 turns) of winding on phase $\mathrm{C}$ of secondary side

In order to evolution of the IECSP modulus oscillations, an index can be defined as the ratio between the maximum and minimum value of the IECSP modulus. Consequently, the extremums ratio (ER) is expressed by:

$$
E R=\frac{\text { maximum of IECSP modulus }}{\text { minimum of IECSP modulus }}
$$

The IECSP locus is illustrated in Fig 6 for a healthy condition and when a turn-to-turn fault involving various shorted turns arises on the different phases of the secondary winding. As seen from the figure, the existence of the inter-turn fault is revealed by disfiguration of the IECSP locus corresponding to a sound situation, leading to a hexagon presentation, whose major axis orientation is pointed to the affected phase and its distortion increases with the fault severity quickly, so that the minor faults are also detectable.

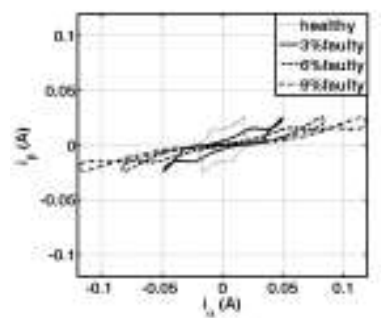

(a)

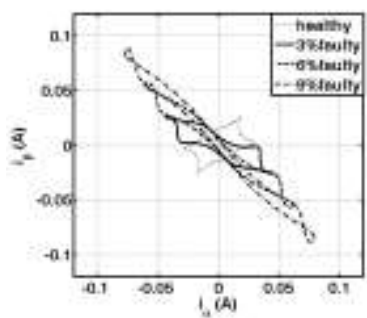

(b)

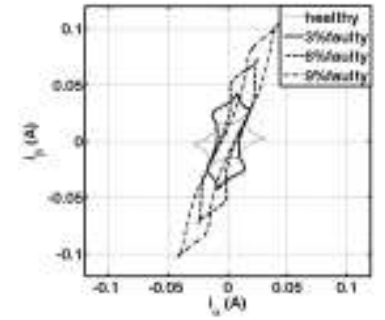

(c)

Fig. 6. Comparison between presentations of IECSP locus in healthy and faulty transformer with several percentages of shorted turns in different phases of secondary winding: (a) phase $\mathrm{A}$; (b) phase $\mathrm{B}$; (c) phase $\mathrm{C}$ 
Under normal condition, the IECSP modulus is close to constant and so contains a dominant DC component as well as a minor AC component that originates of the same reasons accountable for the deformation of the IECSP locus in healthy condition. But in presence of the inter-turn short-circuits the IECSP modulus will oscillate with a frequency twice of the fundamental frequency that its maximum centered on the extremum points of the affected phase exciting current as shown earlier in Fig 6. So, the IECSP modulus will contain a considerable AC component at the frequency of $2 \mathrm{f}$ in addition to a great DC component in a faulty transformer.

Fig 7 exhibits the frequency spectrum related to the IECSP modulus obtained by FFT, under sound situation as well as faulty condition.

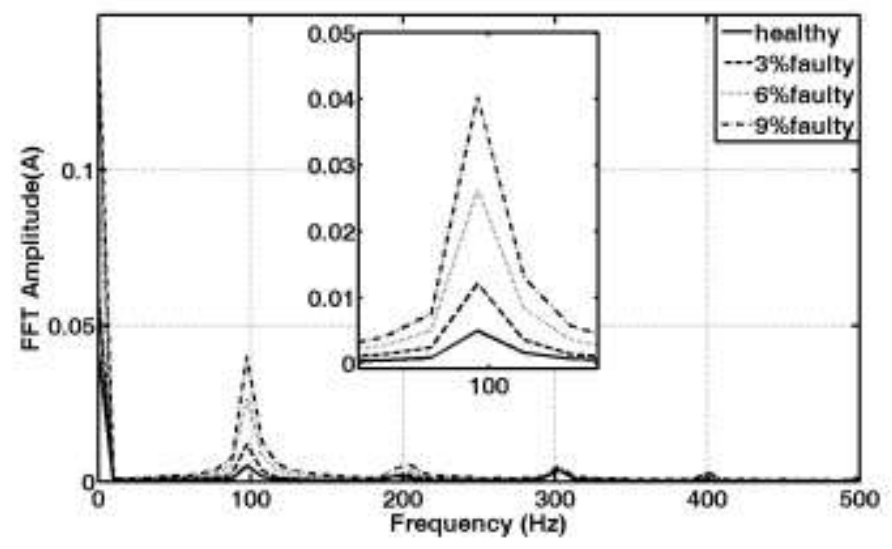

Fig. 7. Frequency spectrum of the IECSP modulus for healthy condition as well as turn-to-turn fault involving several percentages of shorted turns on phase $\mathrm{C}$ of secondary winding

It is observed that the AC component amplitude at the frequency of $2 \mathrm{f}$ is related to the fault intensity, clearly. In this way, similar to [10], the fault severity factor (SF) can be defined by:

$$
\mathrm{S} F=\frac{\left.\left[\sqrt{i_{\alpha}^{2}+i_{\beta}^{2}}\right]\right|_{(2 f) \text { component }}}{\left.\left[\sqrt{i_{\alpha}^{2}+i_{\beta}^{2}}\right]\right|_{\text {DC component }}}
$$

After the fault occurrence, the angular speed of IECSP will be changed, as it is shown in Fig 8. The angle of IECSP is also presented in this figure for different fault severities in phase $\mathrm{C}$ of the secondary winding. In a sound transformer, the IECSP rotates with the angular speed close to constant and its angle changes from 0 to180 degree and vice versa, linearly. While in presence of the inter-turn fault, the angle of IECSP will be changed in a nonlinear manner, so that it is often equal to the affected phase angle (120 and 60 degree) and its angular speed will contain several impulses corresponding to 0 and 180 degree. Obviously, nonlinearity of the IECSP angle and maximum of the angular speed are increased as the fault intensifies. 


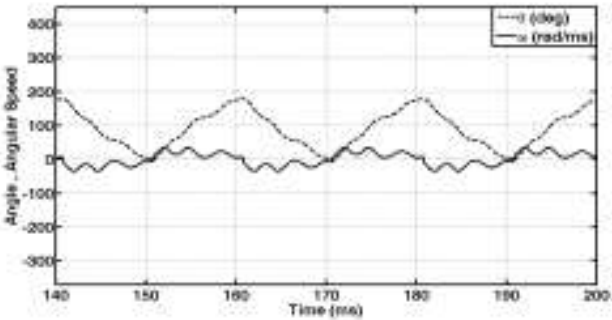

(a)

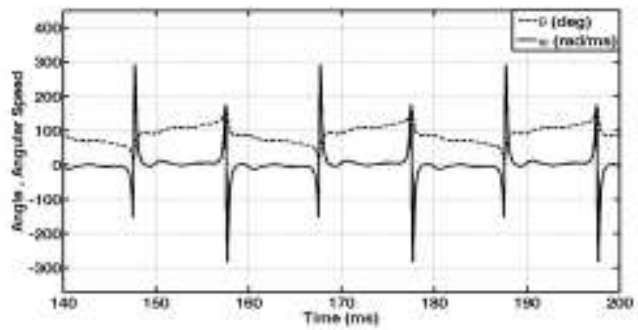

(c)

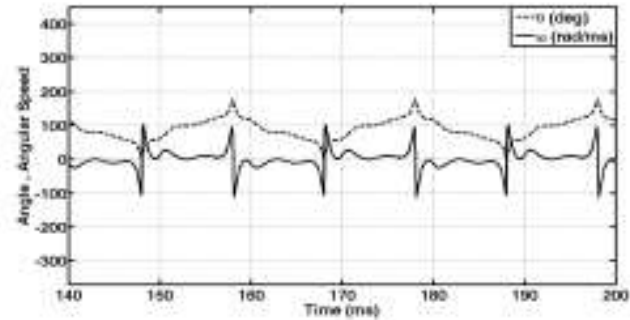

(b)

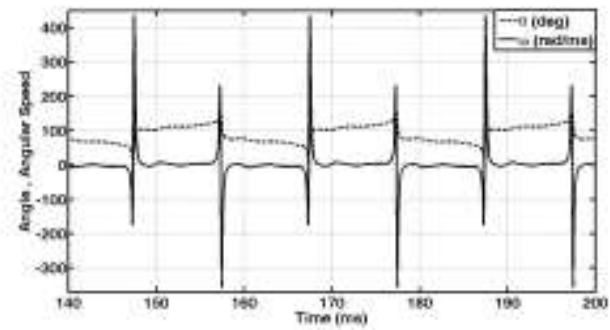

(d)

Fig. 8. Phase and angular speed of IECSP for: (a) healthy condition; and for a turn-to-turn fault involving: (b) $3 \%$; (c) $6 \%$; (d) $9 \%$ of winding on phase $\mathrm{C}$ of secondary side

\subsection{Assessment to the optimum indicator for the IECSP technique}

Some indicators that can be extracted for the proposed technique are listed as below:

- Amplitude of the exciting current in the faulty phase,

- Maximum of the IECSP modulus,

- The extremums ratio,

- The AC component at the frequency of $2 \mathrm{f}$,

- The severity factor,

- Maximum of the angular speed.

In order to assessment to the optimum indicator for the proposed method, the relative increment of the indicators corresponding to growth of the low level faults are calculated and presented in Fig 9. The relative increment is defined as the ratio between the absolute increment and the reference value corresponding to the healthy condition. It should be mentioned that the mean value of IECSP modulus under healthy operation is considered as the reference value for the IECSP modulus indicator.

According to Fig 9 the relative increment associated to the angular speed presents the maximum slope as compared to other graphs. Hence, maximum of the angular speed is most sensitive index and so it can be considered as the optimum index for detection of low level short-circuits. The extremum ratio is another index with proper sensibility that is also more sensitive with regard to the severity factor defined in [10]. 


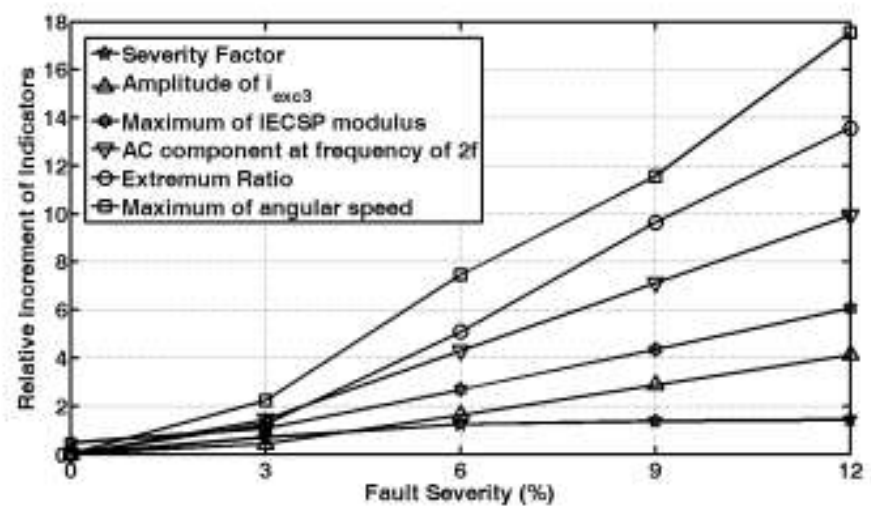

Fig. 9. Relative increment of indicators due to the fault severity in phase $\mathrm{C}$ of secondary winding

\section{Conclusions}

This paper described a new technique that detect the inter-turn short-circuits on the transformer windings by using Instantaneous Exciting Current Space Phasor. The proposed method is based on analyze the exciting currents and hence, it is stable for the case of load imbalance and external faults condition. The occurrence and progression of turn-to-turn faults on the transformer windings had been simulated based on FEM and evolution of the IECSP characteristics with the fault severity had been presented for simulated transformer. It was observed that the propagation of inter-turn fault appears in the deformation of IECSP locus and its stretching toward the faulty phase. As an effect, the oscillation of IECSP modulus with frequency of $2 \mathrm{f}$ and also create impulse in the angular speed waveform of IECSP are symptoms of existence or propagation of winding faults. These characteristics had been used as fault detection tools and some indexes had been extracted from them. The relative increment of these indexes due to growth of the fault had been presented and according to it, the maximum of angular speed had been assessed as the optimum index.

The presented results demonstrate the Instantaneous Exciting Current Space Phasor approach establishes a cost effectiveness online diagnosis technique, that provide fast and reliable detection of low level faults on the transformer windings.

\section{References}

[1] K. L. Butler-Purry and M. Bagriyanik, "Characterization of transients in transformers using Discrete Wavelet Transforms", IEEE Transaction on Power Systems, Volume 18, 2003.

[2] K. L. Butler-Perry and M. Bagriyanik, "Identifying transformer incipient events for maintaining distribution system reliability," in Proceeding the 36th Annual International Conference on System Sciences, Hawaii, 2003.

[3] A. Ashrafian, M. Rostami, G. B. Gharehpetian, and S. S. Shafiee Bahnamiri, "Improving transformer protection by detecting internal incipient faults", International Journal of Computer and Electrical Engineering, Volume 4, 2012.

[4] Q. Zhang, Sh. Jiao, Sh. Wang, "Identification inrush current and internal faults of transformer based on Hyperbolic S-transform", ICIEA, 2009.

[5] R. S. Bhide, M. S. S. Srinivas, A. Banerjee and R. Somakumar, "Analysis of winding inter-turn fault in transformer: a review and transformer models", IEEE ICSET, 2010. 
[6] L. M. R. Oliveira and A. J. M. Cardoso, "On-line diagnostics of transformer winding insulation failures, by Park's Vector approach", Proceedings of the 9th International Electrical Insulation Conference, Berlin, Germany, 2002.

[7] L. M. R. Oliveira and A. J. M. Cardoso, "A coupled electromagnetic transformer model for the analysis of winding inter-turn short-circuits", Record of the IEEE International Symposium on Diagnostics for Electrical Machines, Power Electronics and Drives, Grado, Italy, 2001.

[8] L. M. R. Oliveira and A. J. M. Cardoso, "Intermittent turn-to-turn winding faults diagnosis in power transformers by the On-load Exciting Current Park's Vector approach", Proceedings of the 2008 International Conference on Electrical Machines, IEEE 2008.

[9] L. M. R. Oliveira, A. J. M. Cardoso, S. M. A. Cruz, "Transformers on-load exciting current Park's Vector approach as a tool for winding faults diagnostics", Conference Record of the 15th International Conference on Electrical Machines, Brugge, Belgium, 2002.

[10] L. M. R. Oliveira, A. J. M. Cardoso, S. M. A. Cruz, "Power transformers winding fault diagnosis by the on-load exciting current Extended Park’s Vector Approach”, Electric Power Systems Research 81:1206 1214, 2011.

[11] L. M. R. Oliveira and A. J. M. Cardoso, "Power transformers behavior under the occurrence of inrush currents and turn-to-turn winding insulation faults", XIX International Conference on Electrical Machines, 2010.

[12] A.M. Mahmouda, M.F. El. Naggar, E.H. Shehab Eldina, "A new technique for power transformer protection based on transient components", International Conference on Advances in Energy Engineering, Energy Procedia 14, 2012.

[13] A. Chattopadhyaya, S. Chattopadhyay, S. Sengupta, "Stator current assessment of an induction motor at crawling in Clarke Plane", International Journal of electronics \& communication technology, Volume 4, 2013.

[14] A. Craciunescu, G. Ciumbulea, M. Media, "Stator winding fault diagnostic of induction motor using Instantaneous Currents Space Phasor approach", Prezeglad Electrotechnic (Electrical Review), 2012.

[15] V. Behjat and A. Vahedi, "Numerical modelling of transformers interturn faults and characterising the faulty transformer behaviour under various faults and operating conditions", IET Electrical Power Application, Volume 5, 2011.

\section{Authors Profile}

Malihe Abi was born in Iran, in 1986. She received the B. Sc. and M. Sc. Degrees in Electrical Engineering from University of Birjand, Iran and Babol Noshirvani University of Technology, Iran in 2009 and 2014, respectively. Her research interests include modelling and application of power transfomrers, finite element modelling, and high voltage engineering.

Mohammad Mirzaie was born in Iran in 1975. He obtained B. Sc. and M. Sc. Degrees in Electrical Engineering from University of Shahid Chamran, Ahwaz, Iran and Iran University of Science and Technology, Tehran, Iran in 1997 and 2000 respectively and PhD Degree in Electrical Engineering from Iran University of Science and Technology in 2007. His research interests include high voltage engineering, intelligence networks for internal faults and studying of insulation systems in transformers, cables, generators, insulators, electrical motors. 